\title{
Viabilidad de la informatización del examen lengua extranjera de las Pruebas de Acceso a la Universidad
}

\section{Feasibility of the Computerisation of the Foreign Language Test of the University Entry Exam}

\author{
Ana Sevilla Pavón \\ Universitat de València. España \\ Ana.M.Sevilla@uv.es \\ Ana Gimeno Sanz \\ Universitat Politècnica de València. España \\ agimeno@upv.es \\ Antonio Martínez Sáez \\ Universidad Autónoma de Madrid. España \\ antonio.martinezs@uam.es
}

\begin{abstract}
Resumen
Tras el anuncio por parte del Gobierno de la inclusión de una sección oral en el examen de lengua extranjera de la Prueba de Acceso a la Universidad, que estaba prevista para 2012, diferentes posibilidades en cuanto a la informatización de dicha sección se analizaron en un esfuerzo por encontrar una propuesta viable para la evaluación de las destrezas orales y un estudio cualitativo se llevó a cabo con 286 participantes. Los resultados arrojaron luz en cuanto a la recepción inicial de las soluciones tecnológicas desarrolladas dentro de un sistema de gestión del aprendizaje y en cuanto a la viabilidad de la informatización de la sección oral de la prueba, mostrando que es posible implantar un examen de lengua extranjera asistido por ordenador y adaptado a las características y requisitos anunciados en relación al contenido, la facilidad de uso y la seguridad.
\end{abstract}

\section{Palabras clave}

Destrezas orales; prueba de acceso a la universidad; inglés como lengua extranjera; sistema de gestión del aprendizaje.

\begin{abstract}
Following a Governmental decree announcing the inclusion of oral assessment in the foreign language exam within the Spanish University Entrance Examination to become effective in 2012, the authors set forth to analyse the possibilities of computerizing this exam and design a viable proposal to assess oral skills. To this end, a qualitative study with 286 participants was conducted. The results shed light on the initial acceptance of the technological solutions developed within a dedicated learning management system and on the feasibility of incorporating computerised oral assessment. Evidence that it is indeed possible to implement a computer-assisted foreign language exam adapted to the characteristics and requirements announced with regard to content, usability and security is also provided
\end{abstract}

\section{Key words}

Oral skills; Spanish University Entrance Examination; English as a foreign language; learning management system. 


\section{Introducción}

\section{Introducción}

En los últimos años, el uso de las tecnologías con fines educativos ha venido impulsando cambios importantes en las metodologías de enseñanza y de aprendizaje, proporcionando nuevas herramientas online que pueden ser combinadas con la enseñanza presencial. Existe una gran cantidad de información respecto a diversas de estas herramientas y al modo en que pueden combinarse, la cual es, en muchos casos, proporcionada por profesores a partir de sus experiencias prácticas en el aula (Martín Monje, 2013). Esta valiosa información ha permitido al profesorado actual obtener una serie de directrices muy útiles a la hora de proceder a la utilización efectiva de las Tecnologías de la información y de las comunicaciones (TIC) en sus clases. No obstante, todavía nos encontramos en un proceso de adaptación en el que queda mucho por hacer para que se establezcan y se regulen los parámetros y normas necesarias para garantizar un riguroso control en el uso de estas herramientas, de tal forma que se mantenga la calidad de la enseñanza y de la evaluación de los conocimientos (De Siqueira, Gimeno Sanz, Rego y Amorim, 2010). En este sentido, las Pruebas de Acceso a la Universidad (PAU) no son una excepción, pues dichas pruebas tienen un gran impacto social y son decisivas para el futuro académico y, más tarde, profesional, de los estudiantes que las realizan. De este hecho deriva el gran efecto rebote (washback) que dicho examen produce en el proceso de aprendizaje (Saif, 2006), ya que tanto profesores como estudiantes tienden a centrarse únicamente en los conocimientos y destrezas que el examen evalúa.

El Real Decreto 1892/2008, de 14 de noviembre de 2008, establecía algunos de los cambios que se había previsto introducir. No obstante, dichos cambios se han visto retrasados debido, en gran medida, a la difícil situación que atraviesa nuestro país. Entre los cambios anunciados destaca la inclusión de la evaluación de las destrezas de comprensión y expresión oral en la nueva prueba obligatoria de lengua extranjera de las PAU, que ya no sólo evaluaría las destrezas escritas, como se había venido haciendo en los últimos años, sino que - según lo previsto - pasaría a incorporar, además, la evaluación de las destrezas de comprensión y expresión oral.

A la espera de que los cambios anunciados se hagan efectivos y, con el fin de elaborar una propuesta viable para dar respuesta a los cambios anteriormente señalados, se lanzó el proyecto de investigación nacional Proyecto PAULEX ${ }^{1}$ del grupo de investigación CAMILLE de la Universidad Politécnica de Valencia. El objetivo principal de este proyecto es analizar las posibilidades para la informatización de las pruebas de lenguas extranjeras de las PAU de España. Para alcanzar dicho objetivo, se creó un entorno plurilingüe para la creación, gestión, publicación y corrección de exámenes de idiomas a través de Internet. Este entorno fue desarrollado como un módulo específico que da respuesta a las necesidades concretas de las PAU en España, dentro del sistema de gestión del aprendizaje (SGA) PAULEX. Diversos estudios se llevaron a cabo con el objetivo primordial de poner a prueba las soluciones tecnológicas desarrolladas, atendiendo la creciente necesidad de ofrecer un entorno en línea para la creación,

\footnotetext{
${ }^{1}$ Agradecemos al Ministerio de Ciencia e Innovación del Gobierno de España la concesión y financiación del Proyecto PAULEX (HUM2007-66479-C02-01/FILO), a partir del cual se ha generado este estudio.
}

Viabilidad de la informatización del examen lengua extranjera de las Pruebas de Acceso a la Universidad. Ana Sevilla Pavón, Ana Gimeno Sanz y Antonio Martínez Sáez. 
gestión, publicación y corrección de los exámenes de lenguas extranjera de las PAU. Tal y como puede observarse en las próximas secciones, se prestó especial atención a las soluciones relacionadas con la informatización de pruebas orales, atendiendo aquellas características técnicas más importantes que favorecerían su viabilidad, así como las relacionadas con la operatividad de un examen asistido por ordenador. Posteriormente, se examinaron los resultados del estudio empírico realizado con 286 estudiantes de segundo de bachillerato de centros públicos y concertados de Educación Secundaria Obligatoria de la Comunitat Valenciana. Dicho estudio examinó la acogida inicial, por parte de los estudiantes, de las soluciones tecnológicas desarrolladas en el SGA PAULEX para la realización de la prueba de lengua extranjera (inglés) en las PAU.

\section{Antecedentes en la informatización de exámenes de idiomas}

Algunos de los cambios que se prevé introducir en la sección oral del examen de lengua extranjera de las PAU pueden considerarse una oportunidad para desarrollar herramientas tecnológicas capaces de optimizar el funcionamiento de un examen con las características de la nueva sección de lengua extranjera de las PAU, algo que ya ha ocurrido en mayor o menor medida con otros exámenes de lenguas de gran prestigio e impacto social, tales como el Test of English as a Foreign Language (TOEFL) y el First Certificate in English (FCE). Dicha oportunidad podría darse, por ejemplo, en el caso de la realización de entrevistas virtuales a la hora de evaluar la expresión oral, las cuales serían una alternativa a las entrevistas presenciales que, tradicionalmente, han caracterizado el sistema de evaluación de esta destreza. Asimismo, un número considerable de TIC pueden ser utilizadas para optimizar otros aspectos de las PAU, desde la creación de las pruebas hasta su ejecución y corrección, incluyendo la sección de comprensión y expresión oral. Hoy en día es, además, posible realizar todo ello garantizando altos niveles de seguridad que impedirían que la integridad del sistema se viera afectada. Dos de los ejemplos más destacados que ponen de manifiesto la validez y la viabilidad de una prueba informatizada a gran escala, al menos en lo que a exámenes oficiales de lenguas se refiere, son los sistemas informatizados de pruebas como los anteriormente mencionados TOEFL y FCE.

El TOEFL viene ofreciendo una versión informatizada, el Computer-based TOEFL Test o TOEFL cBT, desde 1998. Además, en septiembre de 2005 se presentó la versión online del examen, el Internet-based TOEFL (TOEFL iBT), que fue introducida inicialmente en Estados Unidos y, posteriormente, en el resto del mundo, de manera progresiva. Los resultados de esta introducción han tenido buena acogida por parte de los usuarios, tal y como reflejan documentos tales como el Test and Score Data Summary for TOEFL® Computer-Based and Paper-Based Tests (Educational Testing Service, 2007). Según los responsables de dicho examen, el TOEFL iBT, que se realiza a través de Internet, evalúa las cuatro destrezas básicas para una comunicación efectiva (comprensión y expresión orales y escritas). Además, el TOEFL iBT enfatiza el uso integrado de las diferentes destrezas y proporciona información referente a las habilidades de expresión oral de los candidatos. El apartado de expresión oral de dicha prueba, que podría servir como ejemplo de cara a plantear un sistema de evaluación de la expresión oral en las PAU, consiste en leer, escuchar y grabar una respuesta de unos 60 segundos de duración a una pregunta determinada. En cuanto a la seguridad, uno de los elementos fundamentales de toda prueba informatizada u ofrecida a través de internet, ésta se cuida al máximo, lo cual se refleja en el hecho de que, entre otras

Viabilidad de la informatización del examen lengua extranjera de las Pruebas de Acceso a la Universidad. Ana Sevilla Pavón, Ana Gimeno Sanz y Antonio Martínez Sáez.

Página 3 de 15 
medidas, se haya establecido que el TOEFL iBT sólo pueda realizarse en los centros autorizados de los distintos países en todo el mundo.

El examen oficial de Cambridge FCE, por su parte, mantuvo el formato tradicional por mucho más tiempo que el TOEFL, pues la implementación de su versión informatizada no comenzó a materializarse hasta el año 2010. En enero de ese año se anunció la informatización del FCE, que pasaba, así, a denominarse Computer-based First Certificate in English Examination (CBFCE). Dicha prueba se sumó, de este modo, a las pruebas oficiales de otros niveles de Cambridge English Language Assessment la Universidad de Cambridge (anteriormente conocida como Cambridge ESOL) que ya se venían realizando desde hacía años a través del ordenador en centros autorizados de todo el mundo (Cambridge English, 2007). Se trata de la versión informatizada del examen oficial de nivel intermedio-alto equivalente al nivel de inglés B2 de acuerdo con el Marco común europeo de referencia para las lenguas (MCER) que evalúa a través del ordenador las destrezas de comprensión y expresión y escritas, y de comprensión oral, pero no de expresión oral (esta última continúa evaluándose de manera presencial). El FCE, que venía realizándose desde hacía varias décadas en el formato tradicional impreso, en combinación con entrevistas personalizadas y tareas colaborativas por parejas para el apartado de expresión oral, ha sido adaptado al formato informatizado para cumplir de manera más eficiente las exigencias actuales de la sociedad global de la comunicación, en que proporcionar la máxima eficiencia y efectividad es indispensable. Sin embargo, a diferencia del TOEFL iBT, el CBFCE no ha presentado una versión en línea, ni permite la evaluación de la destreza de expresión oral a través del ordenador. Por este motivo, a la hora de realizar el FCE se mantienen las entrevistas personales y las tareas colaborativas de candidatos de manera presencial (por parejas), heredadas de las limitaciones del formato impreso.

\section{Posibilidades ofrecidas por PAULEX para la realización de exámenes informatizados}

A pesar de que las PAU se llevan a cabo en todo el territorio nacional, son las consejerías de educación de los diferentes gobiernos autonómicos las encargadas de la gestión de las mismas en cada una de las comunidades autónomas. En el caso concreto de la Comunidad Valenciana, es la Conselleria d'Educació el estamento gubernamental encargado de coordinar, en colaboración con el conjunto de las universidades públicas de la región, las tareas de gestión, diseño, realización y corrección del examen. La labor es facilitada a través de comisiones encargadas de distribuir, custodiar y corregir las pruebas. Todas estas etapas, desde que comienzan a diseñarse los contenidos que se incluirán en la prueba hasta que los alumnos las realizan y finalmente reciben las notas, constituyen un proceso que aún hoy se mantiene en el formato tradicional impreso. El proceso de corrección, realizado a través de tribunales compuestos por profesores de todas las materias que se evalúan en las distintas pruebas, es completamente anónimo, lo cual es posible mediante un sistema de códigos de barras capaz de mantener oculta la identidad del alumno con el fin de garantizar un proceso de corrección, puntuación y revisión lo más objetivo y justo posible. El formato informatizado permitiría conservar estas ventajas del formato actual en cuanto a anonimato y objetividad pero, además, aportaría una serie de ventajas adicionales, que serán detalladas a continuación.

Viabilidad de la informatización del examen lengua extranjera de las Pruebas de Acceso a la Universidad. Ana Sevilla Pavón, Ana Gimeno Sanz y Antonio Martínez Sáez. 
En primer lugar, de cara a la realización y gestión de las PAU y, en concreto, del examen de lengua extranjera, entre las múltiples ventajas de un examen realizado a través del formato electrónico cabe destacar el hecho de que muchas de las tareas y pasos anteriormente descritos se simplificarían. Además, la creación de un sistema de gestión informático robusto permitiría contar con medidas de seguridad férreas y actualizadas, teniendo en cuenta la importancia de estas pruebas de acceso, así como su carácter trascendental para los alumnos que la realizan. Este tipo de sistemas permitiría garantizar, de un modo incluso más estricto y riguroso que en la actualidad, la protección de los exámenes, así como la posibilidad de crear copias de seguridad. Por su parte, el formato electrónico de la prueba facilitaría las labores de gestión y, además, proporcionaría un acceso inmediato, seguro y eficaz a las producciones de los estudiantes por parte de los correctores y posteriores revisores, quienes, a su vez, podrían acceder a un registro con los cambios y modificaciones realizados por cada usuario. En lo concerniente al apartado de expresión oral, que habría de pasar a formar parte del constructo final del examen de lengua extranjera en las PAU, una vez se introduzcan los cambios anunciados, las ventajas de una versión informatizada del examen se verían incrementadas.

En cuanto al nuevo formato de realización de la sección de expresión oral, según la información proporcionada por el Ministerio de Educación (Documento de trabajo del Ministerio de Educación, 2010), éste consistirá en una entrevista por parejas, siguiendo el modo tradicional de examen oral basado en una entrevista a dos que en la actualidad siguen poniendo en práctica organismos examinadores oficiales como los que se encargan de la gestión del anteriormente mencionado Cambridge First Certificate in English. Entre las desventajas de este formato tradicional podríamos citar, como un primer ejemplo, las dificultades para garantizar el anonimato de los examinados, pues el tribunal examinador estaría en contacto directo con los alumnos. Del mismo modo, aspectos como el amplísimo volumen de tiempo que se invertiría en las entrevistas y el enorme esfuerzo humano y económico que supondría la realización de las pruebas siguiendo el formato tradicional hacen que la búsqueda de alternativas sea oportuna. Entre dichas alternativas cabría destacar el sistema de evaluación en línea tratado en el presente artículo, capaz de grabar las respuestas proporcionadas por los alumnos, factor que facilitaría el posterior proceso de revisión en caso de reclamación. La sección de comprensión oral en un formato electrónico también ofrecería claras alternativas de calidad, puesto que cada alumno podría realizar este apartado de la prueba de forma autónoma, independiente y con mayores garantías de calidad de sonido, siendo éste individualizado y de volumen regulable.

El modo de evaluación propuesto forma parte de PAULEX, un SGA que contiene diversas herramientas de evaluación para la realización de exámenes oficiales, como pueden ser las PAU en España. Este sistema surgió para dar respuesta a la necesidad de disponer, a comienzos del siglo XXI, de una herramienta que permitiera a los docentes crear sus propios cursos y materiales didácticos para la enseñanza de lenguas explotando todas las posibilidades multimedia y de transferencia de datos en línea, y que ofreciera a los estudiantes los recursos necesarios para el aprendizaje eficaz de una lengua (Gimeno Sanz, 2006). A este SGA se le han seguido incorporando mejoras sucesivas desde su creación gracias a diversos proyectos de $\mathrm{I}+\mathrm{D}$ en el área del aprendizaje y la evaluación de lenguas asistidos por ordenador, entre los cuales destacan el proyecto europeo CALL@C\&S: online courseware for learners of Czech and Slovak

Viabilidad de la informatización del examen lengua extranjera de las Pruebas de Acceso a la Universidad. Ana Sevilla Pavón, Ana Gimeno Sanz y Antonio Martínez Sáez. 
(114034-CP-1-2004-1-ES-LINGUA-L) ${ }^{2}$ y el proyecto PAULEX, antes mencionado. Concretamente, el proyecto PAULEX impulsó el desarrollo del módulo avanzado para la gestión de exámenes de PAULEX, teniendo en cuenta las características del contexto de las PAU, entre ellas, la gran cantidad de estudiantes que las realizan simultáneamente, así como la importancia de la protección de la información.

Se trata de un SGA innovador, puesto que todos los aspectos de las pruebas son gestionados a través de Internet, incluyendo el desarrollo de los materiales y el control de la interacción entre sus usuarios, los cuales pueden asumir diferentes roles según cada caso, siendo los roles principales "administrador/es del sistema", "autor/es de contenidos", "estudiante/s", "corrector/es y tutor/es", "traductor/es" y "gestores locales"). El sistema funciona con independencia de la plataforma y del ordenador, haciendo uso de los lenguajes y estándares comunes de los navegadores (HTML y JavaScript), por lo que los usuarios pueden utilizarlo desde cualquier parte del mundo con tan solo disponer de un ordenador conectado a Internet. Además, funciona con independencia de otros programas, aunque se requiere la instalación de los plug-ins necesarios para la grabación y reproducción de audio y vídeo. Estructuralmente, se compone de una base de datos interactiva en la que se gestionan los diferentes exámenes y otros materiales, siempre accesibles a través de Internet. Además de las herramientas más comunes de un SGA, como las existentes en ATutor, Dokeos, Moodle, Sakai, OpenUSS o Blackboard, por nombrar algunas; se han incorporado en PAULEX herramientas específicas para el aprendizaje y la evaluación de lenguas extranjeras. Un ejemplo de dichas herramientas específicas lo constituyen las correspondientes al "módulo traductor", las cuales permiten la adaptación de cualquier material a la lengua materna del estudiante, incluyendo la retroalimentación específica para cada ejercicio, facilitando, así, la utilización de componentes culturales en este proceso. También son pioneras las herramientas dedicadas a la evaluación de las destrezas de compresión y de expresión orales del estudiante, tal y como se describirá más adelante. Cabe destacar que se han publicado varios estudios sobre PAULEX desde perspectivas diferentes, tanto a nivel técnico como pedagógico Gimeno Sanz, 2008; Gimeno Sanz, 2006; Gimeno Sanz, de Siqueira, Martínez Sáez \& Sevilla Pavón; 2011; de Siqueira, 2008a).

En el área de evaluación de lenguas asistido por ordenador, especialmente en lo referido a exámenes oficiales con gran transcendencia e impacto social (como es el caso de las PAU), en los últimos años se han venido llevando a cabo diversos estudios sobre distintos aspectos teóricos y técnicos, entre los que destacan:

- Diseño web (García Laborda, 2009);

- El uso de las tecnologías y de los entornos virtuales de aprendizaje en las PAU (García Laborda, Gimeno y de Siqueira,, 2011; Martín Monje, 2012, 2013, 2014);

- Ergonomía (García Laborda, Magal-Royo, de Siqueira y Álvarez, 2010);

\footnotetext{
${ }^{2}$ A través del proyecto CALL@C\&S se crearon cursos en InGenio para el aprendizaje y la evaluación de los idiomas checo y eslovaco para estudiantes europeos. Estos cursos llevaron a la comprobación de que InGenio, en la cual se basa PAULEX, es una plataforma plurilingüe válida para la enseñanza de lenguas que además obtuvo buenos resultados con profesores y estudiantes de toda Europa.
}

Viabilidad de la informatización del examen lengua extranjera de las Pruebas de Acceso a la Universidad. Ana Sevilla Pavón, Ana Gimeno Sanz y Antonio Martínez Sáez. Página 6 de 15 
- Procesos de evaluación en Aprendizaje de Lenguas Asistida por Ordenador (ALAO) (Gimeno, Martínez Sáez, Sevilla Pavón y de Siqueira, 2010; Gimeno, Seiz Ortiz y de Siqueira, 2010);

- Evaluación como proceso intercultural González Pastor, Gimeno Sanz \& de Siqueira, 2010);

- El valor de la retroalimentación informatizada en la evaluación y el aprendizaje (Gimeno Sanz; de Siqueira, 2010 ; de Siqueira 2008b);

- Adaptaciones a otros tipos de dispositivos móviles, como teléfonos móviles o PDA (Giménez López, Magal Royo, Garde Calvo y Prefasi Gomar, 2009);

- La formación en e-learning (Gutiérrez Martín, Palacios Picos y Torrego Egido, 2010; de Siqueira, Gimeno Sanz, Rego y Amorim, 2010);

- Análisis de los costes y de los beneficios en el uso de sistemas informatizados en ALAO (García Laborda, 2009);

- Adaptación de modelos de examen tradicional (formato impreso) a modelos informatizados (de Siqueira, Peris Fajarnes, Giménez y Magal Royo, 2009).

La creación de una herramienta de evaluación en línea como PAULEX pondría a disposición de los usuarios todo tipo de recursos para su utilización en cada una de las fases que hacen posible las PAU, desde la facilidad de edición del examen, para la cual se podría contar con una base de datos y corpus que incorporaran materiales que podrían incluirse como parte del examen; hasta las garantías de éxito a la hora de la realización de las pruebas por parte de una generación de alumnos cada vez más familiarizados con las TIC, las cuales han pasado a formar parte de su vida cotidiana. Así, los estudiantes de bachillerato suelen poseer conexión a Internet tipo aDSL y utilizan el ordenador e internet a diario, siendo en su mayoría autodidactas, todo lo cual hace que se les considere parte integrante de la generación de nativos digitales (Prensky, 2001), capaces de realizar tareas informáticas comunes sin dificultad. Además, los estudiantes consideran tanto el ordenador como Internet una de sus formas de ocio, figurando entre sus actividades favoritas escuchar música, ver vídeos o jugar a videojuegos (Martín Monje, 2013).

La versión informatizada de las pruebas de lenguas extranjeras de las PAU que nos ocupa posee algunas características similares a dos de sus más importantes predecesores, los anteriormente mencionados exámenes FCE y TOEFL, principalmente de este último. Entre dichas características destaca la importancia otorgada a la seguridad, debido tanto al gran número de examinados que realizan la prueba de manera simultánea como al gran impacto que los resultados obtenidos en el examen puede tener en el futuro académico y profesional de los candidatos. También el modo en que se evalúan las destrezas de comprensión oral en la prueba informatizada de las PAU es similar a la manera en que estas destrezas son evaluadas en las dos pruebas antes mencionadas. También el modo en que se evalúan las destrezas de expresión oral en la prueba informatizada de las PAU propuesta se asemeja al modo en que la expresión oral se evalúa en el TOEFL. Así, la evaluación de la expresión oral en PAULEX, al igual

Viabilidad de la informatización del examen lengua extranjera de las Pruebas de Acceso a la Universidad. Ana Sevilla Pavón, Ana Gimeno Sanz y Antonio Martínez Sáez. 
que ocurre con el TOFL IbT, se realiza a través del propio SGA. De este modo, a diferencia de lo que ocurre en el caso del CBFCE, se superan las limitaciones del formato impreso de cara a la evaluación de la expresión oral, lo cual permite que la prueba se beneficie de las ventajas del formato informatizado en cuanto a garantizar la objetividad y el anonimato, junto con la protección de los exámenes y la reducción y facilitación de las labores de gestión, entre otras.

\section{Desarrollo del experimento}

\section{Introducción}

Una de las tareas más importantes en el desarrollo de sistemas informáticos corresponde al análisis de la experimentación con usuarios, en contextos reales o simulados. Este es un proceso fundamental que no solo comprueba si el sistema funciona técnicamente sino que, además, averigua si sus herramientas y los procedimientos son adecuados a las necesidades de sus usuarios. Durante el proceso de desarrollo de PAULEX, se han llevado a cabo diversos experimentos con usuarios, con el fin de optimizar y evaluar sus herramientas. Para este estudio en concreto, que fue llevado a cabo en 2013, se realizó un análisis cualitativo en base a un experimento realizado con 286 participantes. Dicho experimento sirvió para probar el correcto funcionamiento y la efectividad de la plataforma, así como para recopilar datos relativos a la usabilidad y a la satisfacción de sus usuarios, los cuales fueron recogidos a través de encuestas de opinión.

\section{Contexto}

Los usuarios con que se llevó a cabo el experimento eran estudiantes de $2^{\circ}$ curso de bachillerato pertenecientes a tres institutos públicos y tres colegios privados homologados por la Generalitat Valenciana, todos ellos preparándose para presentarse a las PAU en el momento de realización de la experimentación.

Partiendo de la base que los objetivos principales del experimento eran la realización de análisis cualitativos sobre el funcionamiento del SGA para determinar la viabilidad de la informatización de la sección oral de la prueba de lengua extranjera de las PAU, se estableció un procedimiento común seguido por todos los participantes. Con el fin de reducir el tiempo del experimento, el tiempo máximo de realización proporcionado fue de 60 minutos, en lugar de los 90 minutos de realización a los cuales tendrían derecho los estudiantes al realizar las PAU auténticas. Además, se les facilitó a los estudiantes las instrucciones y el apoyo necesario para superar las dificultades relacionadas con la usabilidad del sistema.

Durante la realización de esta simulación, los investigadores realizaron observaciones directas, tanto del funcionamiento del sistema como de las actitudes de los participantes, teniendo en cuenta que las tareas y los procesos pueden afectar en diferente grado a los usuarios (Hawkey, 2009). Cabe destacar que en la mitad de los casos se optó por realizar el experimento en los institutos y escuelas de los estudiantes, para probar el funcionamiento de PAULEX en dichos lugares, dado que es en ellos donde los estudiantes se preparan para las PAU. La otra mitad de los experimentos, en cambio, se realizó en la Universidad Politécnica de Valencia, ya que en ella se realizan cada año las PAU y, por tanto, constituye el entorno real de las pruebas.

Viabilidad de la informatización del examen lengua extranjera de las Pruebas de Acceso a la Universidad. Ana Sevilla Pavón, Ana Gimeno Sanz y Antonio Martínez Sáez. 


\section{Procedimiento}

Partiendo de la premisa de que un análisis de viabilidad comporta un estudio que intenta predecir el eventual éxito o fracaso de un proyecto, para lograr esto partimos de datos empíricos contrastables que fueron extraídos del análisis de cuestionarios. En primer lugar, los estudiantes rellenaron el primero de los dos cuestionarios a través de los cuáles se recopilaron los datos de tipo cualitativo. Dicho cuestionario correspondió a los datos de los participantes, necesarios para tener acceso a aquellos datos personales que pudieran o no influir durante la prueba, entre ellos la edad, el sexo y la experiencia en el manejo de las tecnologías (especialmente, el ordenador e Internet).

Seguidamente, los participantes realizaron, a través de la plataforma, una prueba de inglés correspondiente a la adaptación informatizada de la prueba de inglés de la convocatoria de junio de 2007 de las PAU en la Comunidad Valenciana. Esta adaptación incluyó una propuesta de evaluación de las destrezas orales con contenido multimedia y con grabación de audio. De este modo, las producciones orales de los estudiantes fueron grabadas, con el fin de proporcionar un modelo que se ajustase a las características del nuevo modelo previsto para este examen. Durante este proceso, la observación directa de los investigadores tuvo especial relevancia de cara a cerciorarse de la consecución de los objetivos iniciales.

A continuación, los participantes rellenaron el segundo cuestionario, compuesto por 25 preguntas cerradas, con una escala Likert de 5 puntos (para posterior análisis cuantitativo), relacionadas con la usabilidad del SGA, la facilidad de uso, la facilidad de aprendizaje y la satisfacción global; y 6 preguntas abiertas relacionadas con la utilidad del sistema y la acogida del mismo por parte de los estudiantes, 2 de las cuales inquirían específicamente cuáles eran las expectativas de los estudiantes en cuanto a una futura versión informatizada de las PAU en España. Adicionalmente, se realizó una tercera parte de este experimento con los profesores, quienes posteriormente corrigieron las redacciones y grabaciones de audio de los exámenes de sus respectivos alumnos, lo cual serviría para hacer un análisis cuantitativo que permitiera comparar los resultados obtenidos en la prueba impresa con los obtenidos en la prueba en línea. Además de hacer las correcciones pertinentes, los profesores también respondieron un cuestionario relacionado con los diferentes aspectos relativos a las herramientas de corrección de los exámenes incorporadas en PAULEX.

Durante la realización de la simulación, en la sección de comprensión oral de la prueba, los alumnos visualizaron una serie de vídeos y contestaron varias preguntas formuladas en relación al contenido de éstos. Para responder a estas preguntas, simplemente debían escribir su contestación en los recuadros proporcionados y, después, hacer clic en el botón de "validar", lo cual permitiría que dichas respuestas fueran consideradas como la opción final escogida y, por tanto, como la opción que sería tenida en cuenta de cara al proceso de corrección.

En cuanto a la sección de expresión oral, la evaluación de dicha destreza se llevó a cabo a través de una actividad propuesta a partir de la inclusión de una imagen llamativa sobre un tema de actualidad. Una vez observada la imagen, los participantes debían responder a un enunciado relacionado con el tema sugerido por la misma, exponiendo sus comentarios, opiniones e ideas. Una vez habían pensado lo que querían decir, los participantes grababan su discurso oral de un máximo de 4 minutos, utilizando el micrófono incorporado al ordenador en el que se encontraban realizando la prueba, y el

Viabilidad de la informatización del examen lengua extranjera de las Pruebas de Acceso a la Universidad. Ana Sevilla Pavón, Ana Gimeno Sanz y Antonio Martínez Sáez.

Página 9 de 15 
programa de grabación disponible en el propio sistema. A los estudiantes se les permitía, asimismo, reproducir sus respuestas grabadas para autoevaluarlas e, incluso, volverlas a grabar si no estaban satisfechos con el resultado. Por último, al igual que ocurría en el resto de ejercicios, debían hacer clic en el botón de "validar" para que la versión elegida de sus grabaciones fuera enviada al servidor para su posterior corrección por parte de un tutor.

\section{Análisis e interpretación de las observaciones}

Durante la realización de la prueba por parte de los alumnos, la inmensa mayoría mostró una actitud ejemplar, así como una plena satisfacción con el formato propuesto, siempre sin olvidar que se trata de una generación de nativos digitales (Prensky, 2001), pues son jóvenes acostumbrados a trabajar e interactuar con este tipo de recursos tecnológicos. Por otro lado, cabe señalar varios aspectos de interés observadas durante el experimento, como el hecho anteriormente mencionado de que los alumnos contaran con 60 minutos para la realización de la simulación, en lugar de los 90 minutos de los que dispondrían para la realización de la prueba real. Esta reducción de la duración del examen fue necesaria para interferir lo mínimo posible en sus tareas docentes diarias y no extender el experimento más allá del tiempo de sus clases de inglés. Sin embargo, a pesar de dicha reducción, todos los alumnos lograron finalizar la prueba dentro del espacio de tiempo previsto, completando las actividades relacionadas con la evaluación de todas las destrezas, incluyendo las tareas de comprensión y expresión orales. Los alumnos navegaron por las diferentes partes del examen, escribieron o eligieron sus respectivas respuestas y, seguidamente, validaron sus respuestas, sección por sección. La utilidad de "validación", que se puso a disposición de los alumnos con el fin de que pudieran confirmar sus respuestas de manera más efectiva, ya que las mismas iban quedando guardadas en el servidor para evitar pérdidas de información en caso de cualquier fallo o error que pudiera producirse durante la realización de la prueba, fue muy bien recibida por los estudiantes, hasta tal punto que abundaron los comentarios en relación a la practicidad de la misma.

Uno de los comportamientos que se puso de manifiesto durante la realización de los ejercicios de expresión oral fue el hecho de que los alumnos tendían a escribir un borrador de su respuesta para después leerla y grabarla. Dado que el enunciado del ejercicio no impedía la realización de dicho borrador, los alumnos confesaron que se sentían muchísimo más seguros de su producción si antes podían pensar y reflexionar sobre la respuesta. Esto, naturalmente, le restaba espontaneidad a la respuesta. No obstante, se optó por aceptar estos discursos cuasi preparados porque, si bien es cierto que dichos discursos no eran la forma más adecuada de reflejar las habilidades comunicativas reales de los estudiantes para su posterior evaluación, sí permitían la evaluación de otros aspectos, tales como la pronunciación, la complejidad de las estructuras empleadas, la riqueza de vocabulario y la forma de enlazar las ideas. Este comportamiento es, por otra parte, consistente con la tónica habitual de los alumnos de escribir un borrador del texto para la pregunta de expresión escrita en la actual modalidad de examen de lengua extranjera en formato impreso, con lo que podría considerarse una extrapolación de los comportamientos de expresión escrita a la lengua oral. La fase de experimentación puso también de manifiesto la timidez que la inmensa mayoría del alumnado siente a la hora de expresarse en una lengua extranjera: los alumnos que llegaron a esta parte de la prueba en primer lugar optaban por permanecer en silencio hasta que el resto de sus compañeros también se encontraran en esa misma

Viabilidad de la informatización del examen lengua extranjera de las Pruebas de Acceso a la Universidad. Ana Sevilla Pavón, Ana Gimeno Sanz y Antonio Martínez Sáez.

Página 10 de 15 
sección del examen para así pasar desapercibidos durante la verbalización y grabación. Además, algunos de ellos grabaron con un tono de voz tan bajo que las grabaciones realizadas y escuchadas por los correctores durante la fase de experimentación fueron de muy baja calidad y, a veces, hasta ininteligibles, aspecto que deberá ser tenido en cuenta si finalmente se opta por la informatización de la sección oral del apartado de lengua extranjera de las PAU.

Por otro lado, hubo algunas dificultades técnicas iniciales, que fueron más notables en el caso de las pruebas realizadas en las aulas informáticas de los centros de educación secundaria de los respectivos grupos de alumnos. Afortunadamente, dichas incidencias fueron subsanadas en el caso de los experimentos realizados en las instalaciones de la propia Universidad Politécnica de Valencia (UPV). Se pudo comprobar que, en este caso, características tales como la velocidad de conexión y la calidad de los equipos informáticos permitieron que la experimentación se realizara con una casi total ausencia de dificultades técnicas, a excepción de algunos pequeños fallos relacionados con el volumen del micrófono o con la audición de los archivos producidos por los alumnos antes de validar las respuestas finales en la sección de expresión oral, lo cual haría muy recomendable priorizar la realización de las pruebas informatizadas en universidades similares a la UPV, descartando los centros de educación secundaria o, al menos, aquellos centros que no dispongan de los requisitos técnicos mínimos para la correcta realización de las pruebas informatizadas.

\section{Consideraciones finales}

Los cambios introducidos en la nueva prueba de lengua extranjera de las PAU plantearon la necesidad de hallar posibles soluciones para la implementación de un modo efectivo y viable de evaluar las destrezas orales. Como respuesta a dicha necesidad, se inició el proceso de búsqueda del cual forma parte la fase de experimentación con usuarios descrita en el presente artículo. Hasta la fecha, los estudios que se han llevado a cabo como parte del proyecto PAULEX han mostrado indicios suficientes para mantener el optimismo con el que se inició todo el proceso, el cual quedó patente los resultados del estudio piloto que precedió al aquí descrito (Gimeno Sanz, A., de Siqueira, J. M., Martínez Sáez, A. \& Sevilla Pavón, 2011).

Los resultados del experimento que nos ocupa apuntan que las soluciones y adaptaciones implementadas en PAULEX para la realización de exámenes informatizados de lenguas extranjeras pueden ser consideradas una alternativa válida de cara a la evaluación de la lengua extranjera en las PAU, incluyendo la evaluación de la expresión oral. Así, las pruebas realizadas con alumnos muestran la posibilidad de poner en funcionamiento un examen de lengua extranjera asistido por ordenador adaptado a las características y requisitos específicos de las nuevas PAU. Dichas características y requisitos tienen que ver con las cuestiones de contenidos, usabilidad, y seguridad propias de un examen de tal envergadura, entre otras. En cuanto a las opiniones de los alumnos, éstos se muestran receptivos y no parecen tener mayores dificultades que aquellas que encontrarían al realizar el examen en el formato tradicional impreso.

Todo ello lleva a pensar que el sistema analizado reúne una serie de condiciones que lo hacen adecuado para la preparación previa y la realización de las PAU y, especialmente, de la prueba de lengua extranjera de las mismas. A pesar de ello, para que la implementación de este SGA a gran escala pueda ser llevada a la práctica de manera satisfactoria, es necesario continuar investigando, realizando experimentaciones

Viabilidad de la informatización del examen lengua extranjera de las Pruebas de Acceso a la Universidad. Ana Sevilla Pavón, Ana Gimeno Sanz y Antonio Martínez Sáez.

Página 11 de 15 
similares, tanto en la Comunidad Valenciana como a nivel nacional, de tal manera que la muestra sea mayor y permita, por tanto, la generalización de los resultados ya que, a pesar de que los resultados obtenidos invitan al optimismo, es necesario ser cautos de cara a la generalización de los mismos, puesto que el estudio empírico descrito en el presente artículo se llevó a cabo con tan sólo 286 estudiantes, frente al total de 16.378 alumnos que realizaron las PAU en el año 2013, de acuerdo con los datos proporcionados por el informe del Ministerio de Educación, Cultura y Deporte (2013). Del mismo modo, cabe señalar como limitación del estudio el hecho de que el trabajo se centre en la descripción de la viabilidad del instrumento en cuanto a las posibilidades proporcionadas por la herramienta informática PAULEX. Por ello, los futuros estudios deberán centrarse en analizar las bases teóricas que sustentan el modelo adoptado, en los aspectos metodológicos técnicos relacionados con lo que constituye un análisis de viabilidad y en la efectividad del modelo para la medir el desempeño oral en idioma inglés.

Presentación del artículo: 2 de marzo de 2016

Fecha de aprobación: 9 de marzo de 2016

Fecha de publicación: 30 de abril de 2016

Sevilla-Pavón, A.; Gimeno-Sanz, A. y Martínez-Sáez, A. (2016). Viabilidad de la informatización del examen lengua extranjera de las Pruebas de Acceso a la Universidad. RED. Revista de Educación a Distancia. 49(11). Consultado el (dd/mm/aaaa) en http://www.um.es/ead/red/49

\section{Referencias bibliográficas}

Cambridge English (2007). Boletín de noticias $n^{\circ} 24$ de Cambridge English Exams. Recuperado de http://www.cambridge-exams.ch/newsletter/

Consejo de Europa (2001). Common European Framework of Reference for Languages: Learning, Teaching and Assessment. Cambridge: Cambridge University Press.

De Siqueira, J. M.; Gimeno Sanz, A.; Rego, I. M. S. \& Amorim, J. A. (2010). Algunos dilemas contemporáneos en torno a las tecnologías de la información y de las comunicaciones en la educación: propuesta para la formación de profesores para la producción y el uso de vídeo en el aula, RELATEC, 9(2), 21-35. Recuperado de http://relatec.unex.es/article/view/629

De Siqueira, J. M. (2008a). Integrating Feedback on Language Learning With InGenio. En: Computer-assisted Language Learning (pp. 77-84). Campinas, Sao Paulo: Edições SM.

De Siqueira, J. M. (2008b). La evaluación del aprendizaje en un curso en línea: soluciones del sistema InGenio. En: R. Monroy y A. Sánchez (Eds.), 25 años de lingüística aplicada en España: hitos y retos (pp. 735-741). Murcia: Editum.

De Siqueira, J. M.; Peris-Fajarnes, G.; Giménez, F. \& Magal-Royo, T. (2009). Spanish Students and Teachers' Preferences Towards Computer-Based and Paper-And-Pencil

Viabilidad de la informatización del examen lengua extranjera de las Pruebas de Acceso a la Universidad. Ana Sevilla Pavón, Ana Gimeno Sanz y Antonio Martínez Sáez.

Página 12 de 15 
Tests at Universities. Procedia Social and Behavioral Sciences, 1(1), 814-818.

Recuperado

http://www.sciencedirect.com/science/article/pii/S1877042809001487

de:

Educational Testing Service (2007). Test and Score Data Summary for TOEFL Computer-Based and Paper-Based Tests. Recuperado de http://www.ets.org/Media/Research/pdf/TOEFL-SUM-0506-CBT.pdf

García Laborda, J. (2009). Interface Architecture for Testing in Foreign Language Education. Procedia Social And Behavioral Sciences, 1(1), 2754-2757. Recuperado de: http://www.sciencedirect.com/science/article/pii/S187704280900490X

García Laborda, J.; Magal-Royo, T.; de Siqueira, J. M. \& Álvarez, M. F. (2010). Ergonomics Factors in English As a Foreign Language Testing: The Case of Plevalex. Computers \& Education, 54(2), 384-391. Recuperado de http://www.sciencedirect.com/science/article/pii/S0360131509002164

García Laborda, J., Gimeno Sanz, A. \& de Siqueira, J. M. (2011). Experimentación de las soluciones tecnológicas del proyecto PAULEX para optimizar la prueba de inglés del examen de acceso a la universidad en España. Educação Temática Digital, 12(1), $1-11$.

Gimenez López, J. L., Magal Royo, T., García Laborda, J. \& Garde Calvo, F. (2009). Methods of Adapting Digital Content for the Learning Process via Mobile Devices. Procedia - Social and Behavioral Sciences, 1(1), 2673-2677. Recuperado de http://www.sciencedirect.com/science/article/pii/S1877042809004741

Gimeno Sanz, A., de Siqueira, J. M., Martínez Sáez, A. \& Sevilla Pavón, A. (2011). Paulex Universitas: A Web-Based Management System to Create, Deliver and Assess Online Language Proficiency. En Pixel (Ed.), Proceedings of the $3^{\text {rd }}$ International Conference ICT for Languages Learning. Florencia: Simonelli Editore. Recuperado de http://conference.pixelonline.net/ICT4LL2010/common/download/Proceedings_pdf/IBL03Gimeno_Sanz.pdf

Gimeno Sanz, A. \& De Siqueira, J. M. (2009). Designing Feedback to Support Language Acquisition Using The InGenio Authoring Tool. Procedia Social and Behavioral Sciences, 1(1), 1239-1243. Recuperado de http://www.sciencedirect.com/science/article/pii/S1877042809002249

Gimeno Sanz, A; Martínez Saéz, A.; Sevilla Pavón, A. (2009). Resultados de la encuesta a profesores de $2^{\circ}$ bachillerato para la nueva prueba de lengua extranjera de las PAU LOGSE. Informe interno de la Universidad Politécnica de Valencia. Recuperado de http://www.upv.es/ingles/documentos/informe.pdf

Gimeno Sanz, A. (2006). Proyecto InGenio: gestor de recursos para el aprendizaje de idiomas. Revista de didáctica Marcoele, 2, 1-15. Recuperado de http://marcoele.com/proyecto-ingenio-gestor-de-recursos-para-el-aprendizaje-deidiomas/

Viabilidad de la informatización del examen lengua extranjera de las Pruebas de Acceso a la Universidad. Ana Sevilla Pavón, Ana Gimeno Sanz y Antonio Martínez Sáez. 
Gimeno Sanz, A. (2008). Aprendizaje de lenguas asistido por ordenador: herramientas de autor para el desarrollo de cursos a través de la web. Valencia: Editorial Universidad Politécnica de Valencia.

Gimeno Sanz, A.; Martínez Sáez, A.; Sevilla Pavón, A. \& De Siqueira, J.M. (2010). Student Assessment in the InGenio Online Authoring System: Results Taken from Intermediate Online English. En: R. Durán, S. Sánchez y B. Peñamaría (Eds.), Linguistic Tools for Teachers of English: Towards a Bilingual Education (pp. 51-77). Salamanca: Aquilafuente.

Gimeno Sanz, A.; Seiz Ortiz, R. \& de Siqueira, J. M. (2010). La evaluación del aprendizaje de segundas lenguas a través de la plataforma web InGenio. En: Hacia la Web 3.0 (pp. 101-115). Madrid: Anaya.

Giménez López, J. L., Magal Royo, T., Garde Calvo, F. \& Prefasi Gomar, S. (2009). The Adaptation of Contents for the Creation of Foreign Language Learning Exams for Mobile Devices. International Journal of Interactive Mobile Technologies, (Edición especial), 3, 15-17. Recuperado de http://online-journals.org/ijim/article/view/1008

González Pastor, D.; Gimeno Sanz, A. \& de Siqueira, J. M. (2010). Cultural and technological key considerations of computer assisted language testing in Chinese students learning Spanish. En: New Trends in Computer-assisted Language Learning: Working Together (pp. 117-122). Madrid: Macmillan.

Gutiérrez Martín, A., Palacios Picos, A., \& Torrego Egido, L. (2010). Formar al profesorado inicialmente en habilidades y competencias en TIC: perfiles de una experiencia colaborativa. Revista de Educación, 352, 149-178. Recuperado de http://www.mecd.gob.es/dctm/revista-deeducacion/articulosre352/re35207.pdf?documentId=0901e 72b812342c4

Hawkey, R. A. H. (2009). Examining FCE and CAE: Key issues and recurring themes in developing the First Certificate in English and Certificate in Advanced English exams. Studies in Language Testing, 28. Cambridge: Cambridge University Press and Cambridge ESOL.

Marcelo García, C. (2011). E-learning en la formación para el empleo: ¿qué opinan los usuarios? Revista de Educación, 355, 285-308. Recuperado de http://www.mecd.gob.es/dctm/revista-deeducacion/articulosre355/re35512.pdf?documentId=0901e72b81202ae8

Martín Monje, E. (2014). Integration of Web 2.0 Tools in a VLE to Improve the EFL Spanish University Entrance Examination Results: A Quasi-experimental Study. Calico Journal, 31(1), 19-39. Recuperado de https://journals.equinoxpub.com/index.php/CALICO/article/view/22801

Martín Monje, E. (2013). El aprendizaje mixto en la preparación de la Prueba de Acceso a la Universidad en Lengua Inglesa: Aportaciones de un estudio experimental. Porta

Viabilidad de la informatización del examen lengua extranjera de las Pruebas de Acceso a la Universidad. Ana Sevilla Pavón, Ana Gimeno Sanz y Antonio Martínez Sáez. 
Linguarum, $\quad$ 19, 71-85. Recuperado de http://www.ugr.es/ portalin/articulos/PL_numero19/5\%20Elena\%20Martin.pdf

Martín Monje, E. (2012). El nuevo examen oral en la Prueba de Acceso a la Universidad en lengua inglesa. Una propuesta metodológica. Revista de Educación, 357, 143-161. Recuperado de http://www.revistaeducacion.educacion.es/re357/re357_07.pdf

Ministerio de Educación, Gobierno de España (2013). Datos y cifras del sistema universitario español. Recuperado de http://www.mecd.gob.es/dms/mecd/educacionmecd/areas-educacion/universidades/estadisticas-informes/estadisticas-informesdocumentum/datos-cifras/2012-2013-datos-y-cifras-sistema-universitarioespanol.pdf

Ministerio de Educación, Gobierno de España (2010). Características específicas de la prueba oral del ejercicio de lengua extranjera de la PAU. Secretaría de Estado de Educación y Formación Profesional; Dirección General de Evaluación y Cooperación Territorial.

Ministerio de Educación, Gobierno de España (2008). Real Decreto 1892/2008, de 14 de noviembre, por el que se regulan las condiciones para el acceso a las enseñanzas universitarias oficiales de grado y los procedimientos de admisión a las universidades públicas españolas.

Prensky, M. (2001). "Digital Natives, Digital Immigrants", On the Horizon, 9(5), 1-6. Recuperado http://www.emeraldinsight.com/doi/pdfplus/10.1108/10748120110424816

Saif, S. (2006). Aiming for positive washback: A case study of international teaching assistants. Language Testing, 23(1), 1-34. Recuperado de http://ltj.sagepub.com/content/23/1/1.full.pdf+html

\section{Agradecimientos}

Los autores desean mostrar su agradecimiento al Ministerio de Ciencia e Innovación del Gobierno de España por la concesión y financiación del proyecto PAULEX (HUM2007-66479-C02-01/FILO), a partir del cual se ha generado este estudio. 